\title{
Non-Integrable Dynamics and Physics Beyond the Standard Model
}

\author{
Ervin Goldfain \\ Research Scholar, Ronin Institute, Montclair, New Jersey 07043 \\ Email: ervin.goldfain@ronininstitute.org
}

\begin{abstract}
The evolution of integrable classical systems leads to conserved quantities and vanishing Poisson brackets. In contrast, such invariants do not exist in the dynamics of non-integrable systems, which include (but are not limited to) deterministic models with long-term chaotic behavior. The object of this review is to briefly survey the mathematical background of nonintegrability and its role in the physics unfolding well-above the Standard Model (SM) scale.
\end{abstract}

Key words: Non-integrability, Poincaré resonances, fractional dynamics, Haag's theorem, interacting Quantum Field Theory, Beyond-the-Standard-Model physics.

\section{Introduction}

A cornerstone postulate of perturbative Quantum Field Theory (QFT) is the cluster decomposition principle (CDP), according to which local processes are insensitive to distant environments [1]. In a nutshell, CDP allows one to isolate a subsystem from all others that are widely separated in either spacetime or energy scale. In doing so, CDP ultimately secures consistency of both Renormalization Group and the perturbative formulation of QFT. 
One may reasonably ask if CDP survives or is ruled out somewhere above the SM scale. There are well motivated reasons to suspect that the deep Terascale sector prevents thermalization of quantum fluctuations and creates an environment favoring the onset of complex dynamics [2]. In this setting, the underlying principles of classical statistical physics and perturbative QFT are likely to break down. The ergodic theorem, the fluctuation-dissipation theorem, analyticity, unitarity, locality, finiteness in all orders of perturbation theory and renormalizability are either violated or lose their conventional meaning.

It is known that, while the dynamics of integrable classical or quantum systems leads to conserved quantities, such invariants do not generally exist for non-integrable systems. The goal of this report is to sketch the plausible connection between non-integrability of phenomena lying well above the SM scale and the breakdown of CDP.

\section{Poincaré resonances and non-integrability}

Non-integrable dynamics (in the Poincaré sense) arises from adding interaction terms to free Hamiltonian systems. In general, the presence of such terms entails a destruction of the invariants of motion [3-4].

This can be seen from a straightforward example. Consider a classical Hamiltonian having the form

$$
H=H_{0}+\lambda V
$$

including a perturbation of strength $\lambda$ added to the free term $H_{0}$. The invariants of motion $\Phi^{0}$ for the free system ( $\lambda=0$ ) follow from the vanishing Poisson bracket 


$$
\left\{H_{0}, \Phi^{0}\right\}=0
$$

Integrable systems are characterized by a set of invariants of motion $\Phi=\Phi(\lambda)$ that are analytic in $\lambda$ and yield a vanishing Poisson bracket,

$$
\{H, \Phi\}=0
$$

Typical examples of integral invariants include the generalized momenta derived from the Hamilton-Jacobi equation of classical mechanics [5]. By contrast, such invariants $\Phi$ are missing from systems that are non-integrable. To understand why this is the case, we start from (2.3), expand $\Phi$ in power series

$$
\Phi=\sum_{n} \lambda^{n} \Phi^{n}
$$

and develop each of the terms $\Phi^{n}$ in Fourier series to arrive at

$$
\{H, \Phi\}=\left\{H_{0}, \Phi^{n}\right\}+\left\{V, \Phi^{n-1}\right\}=0
$$

It can be shown that (2.5) is equivalent to vanishing of all Fourier coefficients $\varphi_{k}^{0}=0$ corresponding to wavevectors $k$. Non-integrable systems fail to satisfy (2.5) in resonance conditions, whereby $\varphi_{k}^{0} \neq 0[4]$.

We next show that a similar situation occurs in Quantum Mechanics. Iterating the arguments of [6], we start with the assumption that the eigenvalue problem for the $H_{0}$ operator is solved and that its eigenvalue spectrum is given by the discrete sequence $E_{i}^{0}, i=1,2, \ldots$ The spectrum of $H=H(\lambda)$ is a continuous function of $\lambda$ such that 


$$
H(0)=H_{0}
$$

Let $E$ denote a non-degenerate eigenvalue of $H$ that fulfills the condition

$$
E(\lambda=0)=E_{a}^{0}
$$

in which $E_{a}^{0}$ represents a given eigenvalue of $H_{0}$. The corresponding eigenvector $|\psi\rangle$ is defined by

$$
H|\psi\rangle=E|\psi\rangle
$$

along with the following requirements

$$
\begin{gathered}
\langle 0 \mid \psi\rangle=\langle 0 \mid 0\rangle=1 \\
|\psi\rangle \stackrel{\lambda=0}{\longrightarrow}|0\rangle
\end{gathered}
$$

The small perturbation approximation $\left(\lambda V<<H_{0}\right)$ justifies expanding $E$ and $|\psi\rangle$ into the power series

$$
\begin{gathered}
E=E_{a}^{0}+\lambda \varepsilon_{1}+\lambda^{2} \varepsilon_{2}+\ldots+\lambda^{n} \varepsilon_{n}+\ldots \\
|\psi\rangle=|0\rangle+\lambda|1\rangle+\lambda^{2}|2\rangle+\ldots+\lambda^{n}|n\rangle+\ldots
\end{gathered}
$$

It can be shown that the $n^{\text {th }}$ order correction to the eigenvector $|\psi\rangle$ in (2.12) may be expressed as

$$
|n\rangle=\frac{Q_{0}}{a}\left[\left(V-\varepsilon_{1}\right)|n-1\rangle-\varepsilon_{2}|n-2\rangle-\ldots-\varepsilon_{n-1}|1\rangle\right]
$$


in which

$$
\begin{aligned}
& Q_{0}=\sum_{E^{0} \neq E_{a}^{0}} \sum_{\alpha}\left|E_{\alpha}^{0}\right\rangle\left\langle E_{\alpha}^{0}\right| \\
& \frac{Q_{0}}{a}=\sum_{E^{0} \neq E_{a}^{0}} \frac{\sum_{\alpha}\left|E_{\alpha}^{0}\right\rangle\left\langle E_{\alpha}^{0}\right|}{E_{a}^{0}-E^{0}}
\end{aligned}
$$

To fix ideas, consider the case $n=1$. Equation (2.13) gives

$$
|1\rangle=\frac{Q_{0}}{a}\left(V-\varepsilon_{1}\right)|0\rangle
$$

or, since $Q_{0}|0\rangle=0,(2.16)$ amounts to

$$
|1\rangle=\frac{Q_{0}}{a} V|0\rangle
$$

Thus, the first-order correction to $|\psi\rangle$ takes the form

$$
|\psi\rangle=\left(1+\lambda \frac{Q_{0}}{a} V\right)|0\rangle+O\left(\lambda^{2}\right)
$$

By (2.17), the components of the first-order correction to $|0\rangle$, measured along all the other basis vectors of $H_{0}$ are

$$
\lambda\left\langle E_{\alpha}^{0} \mid 1\right\rangle=\frac{\left\langle E_{\alpha}^{0}|(\lambda V)| 0\right\rangle}{E_{\alpha}^{0}-E^{0}}
$$


Relation (2.19) states that the component along $\left|E_{\alpha}^{0}\right\rangle$ is equal to the perturbation matrix element connecting $|0\rangle$ and $\left|E_{\alpha}^{0}\right\rangle$ divided by the energy difference between these two unperturbed eigenstates. The magnitude of (2.19) quantifies the rate at which the perturbation series converges. It is readily seen that (2.19) diverges in resonance conditions defined by $E_{\alpha}^{0}=E^{0}$.

\section{Final remarks}

Historically, Prigogine and his Brussels-Austin school of non-equilibrium thermodynamics were the first to point out that, under certain conditions, Poincaré resonances can render Quantum Mechanics inconsistent and deny its celebrated predictivity $[7-8,15]$. Few observations are required to further clarify this point:

3.1) Singular denominators are also present when computing scattering amplitudes in perturbative QFT. One must bear in mind that, while the so-called is prescription of QFT evades singularities by isolating the propagator poles [1, 9], Poincaré resonances describe persistent scattering, a genuinely non-perturbative process falling outside the boundaries of conventional QFT. By coupling the creation and annihilation operators, Poincaré resonances generate time irreversibility and nonlocality at the quantum level of observation [15].

3.2) A tacit assumption of all covariant field theories is that coordinate and gauge transformations, along with their inverse, are well-behaved functions that can be differentiated arbitrarily many times. But this assumption fails to be true in general due to the broad spectrum of existing non-differentiable functions and trajectories, 
as repeatedly discovered since the introduction of fractal geometry in 1983. Many authors have emphasized that fractional calculus and fractional dynamics provide the adequate framework for the analysis of such objects [10-14].

3.3) Unlike conventional dynamics on smooth manifolds, fractional dynamics implies nonlocality, dissipation, and power-law correlations [10-14]. These attributes are inherently linked to the mixing of ultraviolet and infrared sectors above the SM scale and lead to the violation of the CDP postulate. Indeed, the formulation of CDP stems from the assumption of Gaussian statistics and Central Limit Theorem, which likely break down in non-equilibrium dynamical settings $[2,10]$.

3.4) One recalls that the rigorous construction of interacting QFT is confronted by specific challenges brought up by the Haag's theorem [1]. This theorem asserts that the quantum interaction picture breaks down when the free-field Hamiltonian $\left(H_{0}\right)$ and its interacting part $(V)$ differ from each other by the integral of some local operator density. The prevailing view is that the Haag theorem can be circumvented by full regularization of QFT, in which both a spatial infrared cutoff (that is, a finite spatial volume) and an ultraviolet cutoff (that is, a finite lattice spacing) are introduced. It is unclear, however, if evading the Haag theorem by regularization survives a dynamical regime where non-integrability sets in and CDP no longer holds.

\section{References}

1. Duncan A., "The Conceptual Framework of Quantum Field Theory", Oxford Univ. Press 2012. 
2. Available at the following sites:

https://www.researchgate.net/publication/344227402_Complex_Dynamics_and_Fou ndational Physics

Goldfain, E. Complex Dynamics and Foundational Physics. Preprints 2020, 2020110263

3. https://arxiv.org/pdf/cond-mat/ooo1198.pdf

4. Gutzwiller M. C., "Chaos in Classical and Quantum Mechanics”, Springer-Verlag 1990.

5. Landau L. and Lifshitz, E, "Mechanics", Course of Theoretical Physics, vol. 1 Butterworth-Heinemann 1976.

6. Messiah A., "Quantum Mechanics”, Dover Edition, 1999.

7. https://www.pnas.org/content/pnas/90/20/9393.full.pdf

8. https://onlinelibrary.wiley.com/doi/abs/10.1002/qua.560530115

9. https://physics.weber.edu/schroeder/qftbook.html

10. West B.J., Bologna M. and Grigolini P., "Physics of Fractal Operators", Springer 2003.

11. https://www.sciencedirect.com/science/article/abs/pii/S1007570406001183

12. Goldfain E., "Fractional Field Theory and High-Energy Physics: New Developments", International Journal of Theoretical Physics, Group Theory, and Nonlinear Optics; Vol. 16, Issue 3/4, 2012, pp. 239-262.

13. Available at the following site:

https://www.researchgate.net/publication/275154184 On Nonintegrability and the Asymptotic Breakdown of Perturbative Field Theory

14. Available at the following site:

http://www.aracneeditrice.it/aracneweb/index.php/pubblicazione.html?item=9788854 $\underline{889972}$

15. Prigogine I., "The End of Certainty", The Free Press 1997. 